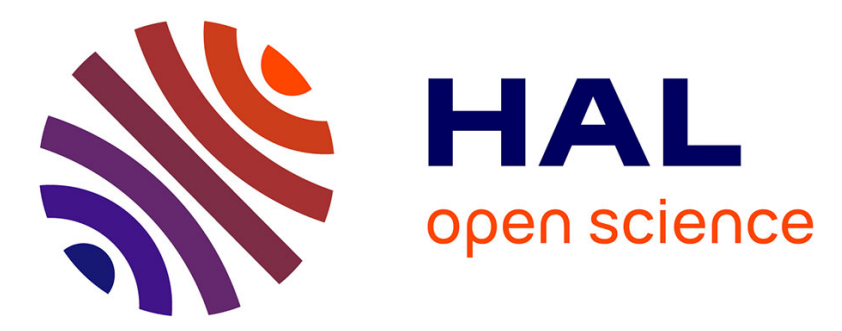

\title{
Les moules monétaires de Corseul (Côtes-d'Armor) et la date de fabrication des faux deniers en Gaule
}

Gérard Aubin

\section{To cite this version:}

Gérard Aubin. Les moules monétaires de Corseul (Côtes-d'Armor) et la date de fabrication des faux deniers en Gaule. Gallia - Fouilles et monuments archéologiques en France métropolitaine, 1990, 47, pp.257-263. 10.3406/galia.1990.2911 . hal-01918322

\section{HAL Id: hal-01918322 \\ https://hal.science/hal-01918322}

Submitted on 20 Jan 2020

HAL is a multi-disciplinary open access archive for the deposit and dissemination of scientific research documents, whether they are published or not. The documents may come from teaching and research institutions in France or abroad, or from public or private research centers.
L'archive ouverte pluridisciplinaire HAL, est destinée au dépôt et à la diffusion de documents scientifiques de niveau recherche, publiés ou non, émanant des établissements d'enseignement et de recherche français ou étrangers, des laboratoires publics ou privés.

\section{(1) (1) $\$$}

Distributed under a Creative Commons Attribution - NonCommercial - NoDerivatives| 4.0 


\title{
LES MOULES MONÉTAIRES DE CORSEUL (CÔTES-D'ARMOR) ET LA DATE DE FABRICATION DES FAUX DENIERS EN GAULE
}

\author{
par Gérard AUBIN
}

Trois moules monétaires en terre cuite, conservés au musée de Nantes, proviennent de Corseul. Deux reproduisent des deniers sévériens. Le troisième présente l'empreinte d'un droit de denier de Julia Domna (196211) et, sur l'autre face, celle du revers d'un antoninianus d'Aurélien (Milan, fin 271-été 273). La production de faux deniers coulés sous Aurélien, alors que cette espèce n'était pratiquement plus frappée, amène à retenir l'hypothèse de faux-monnayeurs mettant à profit la désorganisation de la circulation monétaire pour tromper les thésaurisateurs. On doit admettre que le denier avait acquis un cours parallèle et officieux, croissant au fur et à mesure de la dégradation de l'antoninianus.

Il est possible que les lots de moules datés de l'époque sévérienne soient en fait le produit d'une activité frauduleuse plus tardive (vers 265-305) contemporaine de la frappe d'abondantes imitations radiées. Le relevé des monnaies coulées dans les trésors tend à confirmer cette hypothèse.

Three clay moulds coming from Corseul are conserved in Nantes museum. Two of them represent severian denarii. The third mould shows the print of a denarius obverse of Julia Domna (196-211), and on the other side, of an antoninianus reverse of Aurelian (Milan, late 271-summer 273). The production of cast denarii in the time of Aurelian, when the coinage of denarii had practically ceased, suggests the hypothesis of counterfeiters who took advantage of the disorganization of the monetary circulation to mislead hoarders. We must admit that the denarius had reached a parallel and unofficial rate, increasing in proportion with the debasement of the antoninianus.

The series of severian coin-moulds may be the product of a late fraudulent activity (v. 265-305) contemporaneous with the manufacture of "barbarous radiates". A conspectus of casts found in hoards seems to confirm the hypothesis.

Le classement des collections numismatiques des musées départementaux de Loire-Atlantique ${ }^{1}$ m'a fait découvrir trois moules monétaires en terre cuite, sur le rebord desquels on avait porté à l'encre noire la mention "Corseul». Ces objets ne figurent pas dans les catalogues du musée ni dans les registres d'entrées manuscrits tenus à jour jusqu'en 1903. La bibliographie consultée sur Corseul, chef-lieu de la

1 Je remercie M. Jacques Santrot, Conservateur des musées départementaux, qui m'a facilité l'accès à ces documents. civitas des Coriosolites n'est d'aucun secours ${ }^{2}$. On ignore donc tout de l'endroit, de la date et des circonstances de cette trouvaille. Le fait que les érudits nantais ayant eu à s'occuper de moules

2 G. Guennou, La Cité des Coriosolites, Dossiers du Centre Régional Archéologique d'Alet, n D, Saint-Malo, 1981, 177 p. ; - L. LANGOUËT, Les Coriosolites, un peuple armoricain de la période gauloise à l'époque gallo-romaine, Saint-Malo, Centre Régional d'Archéologie d'Alet, 1988, p. 218 : l'auteur en fait une brève mention à la suite de l'information orale que je lui ai donnée. 
monétaires ${ }^{3}$ n'en fassent pas état laisserait présumer que ces objets sont entrés au musée postérieurement à 1910 .

\section{CATALOGUE}

Les empreintes des moules ont le module du denier (diamètre du grènetis : 17,7 à 18,5) sauf l'empreinte $3 \mathrm{~B}$ qui est celle d'un antoninianus (diamètre du grènetis : 22,2). Le catalogue suit les normes proposées par R. Turcan dans la publication des moules du site lyonnais du "Verbe-Incarné»: la description des légendes, effigies et types correspond à celle des monnaies utilisées pour les empreintes ou des épreuves issues des moules.
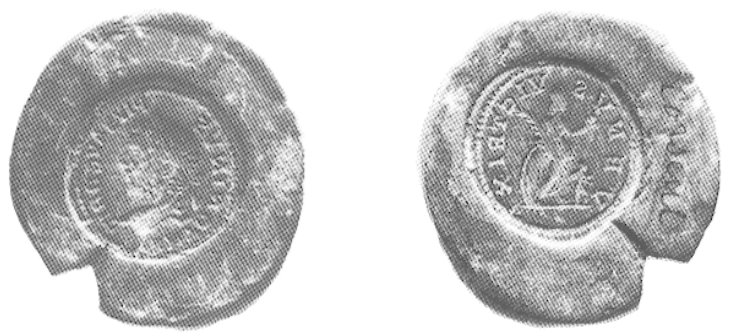

1 Pâte brun-rouge et grise (diamètre : 31,5-32 mm; épaisseur : 8-9,6 mm); rainure à $7 \mathrm{~h}$. au droit et à $5 \mathrm{~h}$. au revers.

A/ ANTONINVS - PIVS AVG BRIT, busle lauré et drapé à droite; effigie de Caracalla et légende des années 210-213 : RIC, IV-1, p. 62,85 ; 237245.

B/ VENVS VICTRIX, Vénus debout à gauche, s'appuyant sur un bouclier et tenant une palme et une pomme; à ses pieds, à gauche, Cupidon. Type d'un denier de Plautille : $R I C$, IV-1, n 369 (Rome, 202-205).
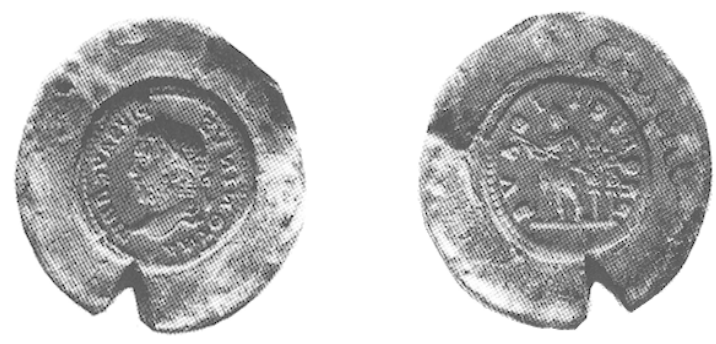

2 Pâte grise avec des parties noirâtres (diamètre :

3 L.-J.-M. Bizeul, Des moules monétaires, Bulletin de la Sociélé Archéologique de Nantes et de la Loire-Inférieure, I, 1859-1861, p. 385-394: “il n'en a pas encore été trouvé en Bretagne" (p. 385); - P. Sovi.t.ARD, Un mot sur les moules de monnaies romaines trouvés à Rezé, ibid., L, 1909, p. 201-206.
30,5-31 $\mathrm{mm}$; épaisseur : 7,4-9,4 $\mathrm{mm}$ ); rainure à $7 \mathrm{~h}$. au droit et $5 \mathrm{~h}$. au revers.

A/ Même effigie que 1A.

B/ PROFECTIO - AVG, Caracalla s'avançant à droite, un javelot à la main; derrière lui, deux enseignes. Type d'un denier de Caracalla : $R I C$, IV-1, p. 244, no 225 (Rome, 210-213).
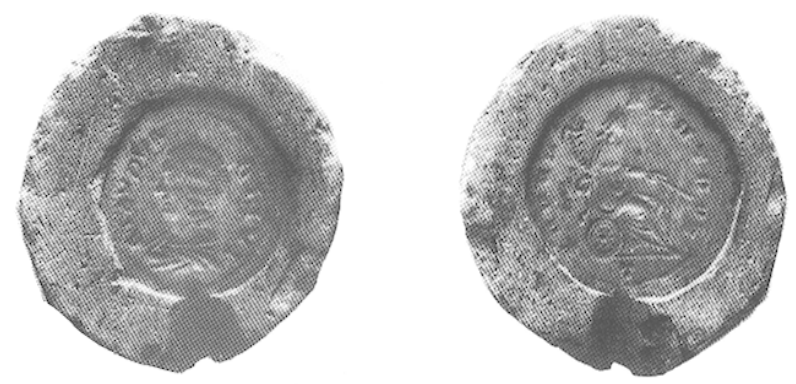

3 Pâte beige rosé clair (diamètre $34,7-35 \mathrm{~mm}$; épaisseur : $6,6 \mathrm{~mm}$ ); rainure à $6 \mathrm{~h}$. au droit et au revers.

A/ IVLIA - AVGVSTA, buste drapé à droite; effigie de Julia Domna et légende des années 196$211: R I C$, IV-1, p. 63, 166 et $s q$.

B/ FO-RTVNA REDUX, T à l'exergue, Fortuna assise à gauche sur une roue, tenant un gouvernail et une corne d'abondance. Revers d'un antoninien d'Aurélien (Milan, $2^{\mathrm{e}}$ ou $3^{\mathrm{e}}$ émission, 271-273) : RIC V-1, p. 279, no 128; - S. Estiot, Le trésor de Maravielle (Var), Trésors monétaires, $\mathrm{V}, 1983$, p. 19 et catal. $\mathrm{n}^{\text {os }} 168,202,261-273$.

Ces trois moules ne peuvent être que le reliquat d'un lot plus important. En effet, on connaît l'agencement de ces rondelles de terre cuite depuis les découvertes de Damery (Marne), de Pachten (Sarre) ou encore de Qasr-Qarum (Egypte) ${ }^{4}$. Le principe en a été rappelé récemment par R. Turcan ${ }^{5}$ : les tablettes d'argile portant les empreintes de deux monnaies différentes (ordinairement un droit et un revers) étaient disposées en pile, de telle façon qu'une empreinte de droit corresponde à une empreinte de revers. Sur chaque face du moule, une rainure était ménagée pour permettre l'écoulement du métal en fusion. Les rainures de

4 A. Hiver, Notice sur un atelier monétaire découvert à Damery (Marne) en 1830, Revue Numismatique, 1837, p. 171180, pl. VI ; - M.-R. Alfoeldi, Die Gussformen und gegossenen Fälschungen Kaiserzeitlicher Münzen, Chiron, 1, 1971, p. 351-363; - J. Schwartz, H. WILd, Qasr-Qarum / Dionysias 1948 (Fouilles franco-suisses, Rapports, I), Le Caire (Publications de l'I.F.A.O. du Caire), 1950, p. 39-48, pl. XII.

5 R. Turcan, Les moules monétaires du Verbe Incarné (Lyon), Trésors Monétaires, IV, 1982, p. 9-29, pl. I-V (p. 1011). 

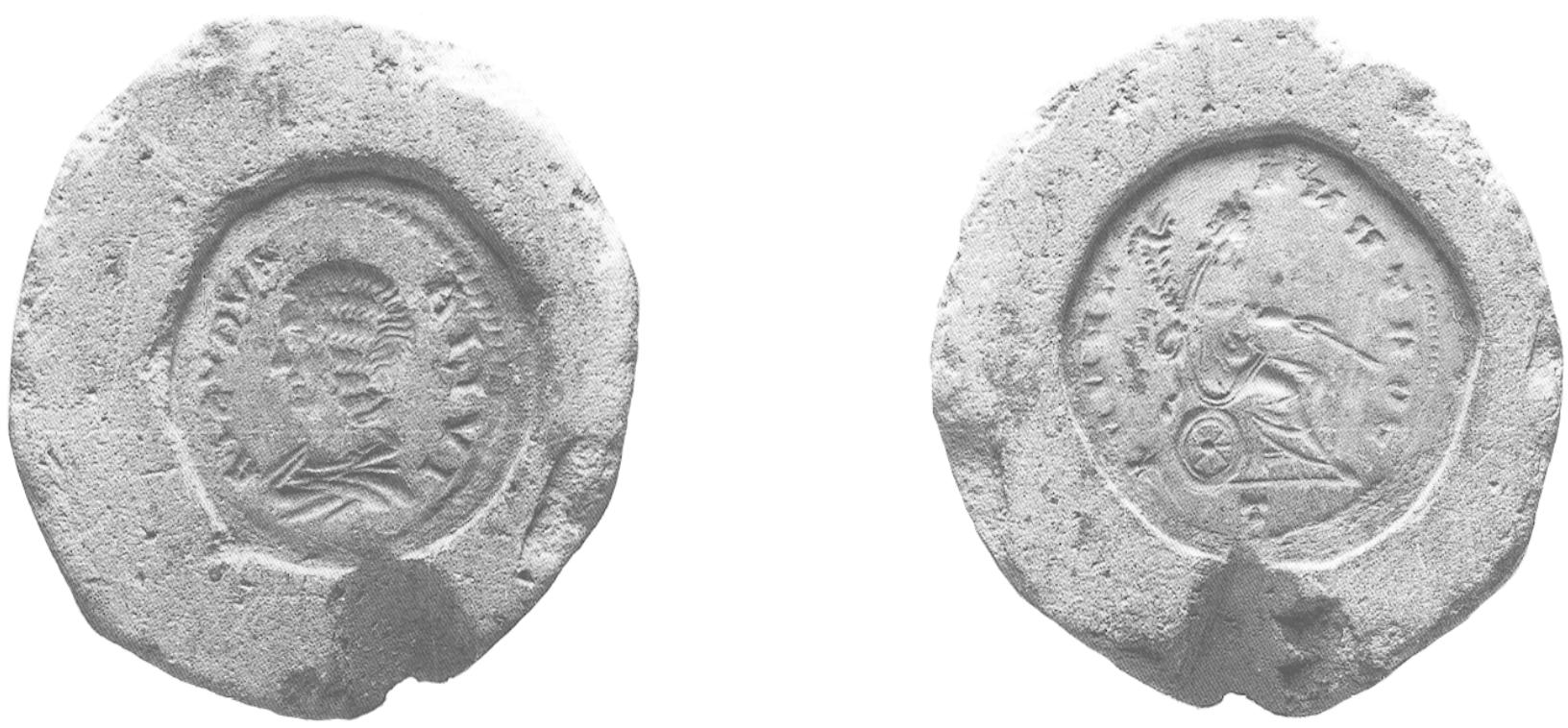

Fig. 1 - Moule monétaire no 3 (au double de sa grandeur).

l'ensemble des moules de la pile étaient reliées par une entaille formant gouttière. Les rouleaux composés de huit à treize moules pouvaient être réunis par trois, l'ensemble étant enveloppé d'argile molle. Ainsi une seule coulée permettait de fabriquer vingt et une ou trente-six monnaies. La récupération des matrices était délicate et beaucoup devaient être brisées au cours du démoulage.

Les trois moules de Corseul appartiennent à trois piles différentes car les entailles de coulée, de taille variable, ne se superposent pas. Toutefois, rien n'interdit qu'ils proviennent d'un même cylindre de trois rouleaux, c'est-à-dire qu'ils aient servi lors d'une même coulée. Leur état de conservation est excellent; les empreintes, très lisibles, ont été obtenues avec des monnaies ayant peu circulé. Leur pâte varie du rouge-jaune au brun avec des zones grisées dues à la combustion (notamment le $n^{\circ} 2$ ). Ils semblent avoir peu servi. Par ailleurs, à l'opposé de l'entaille réservée au passage du métal, la tranche est incisée d'un trait vertical sur les $n^{\text {os }} 1$ et 2 , d'un trait vertical et de deux traits horizontaux sur le $n^{0} 3$. Il s'agit sans doute de repères pour l'ajustement des moules lors de leur réutilisation.

L'intérêt majeur de cette trouvaille est chronologique. Certes, la reproduction par moulage de deniers des années 196-213 (pour trois droits et deux revers) n'est pas pour surprendre. Les deniers notamment sévériens - sont en effet l'espèce de prédilection des faux-monnayeurs en Allemagne, en Grande-Bretagne et dans une moindre mesure en France où "le procédé continue d'être employé, pour des antoniniani, quelquefois mêlés à des deniers, jusque sous Tetricus, en $273 \|^{6}$. Il est toujours délicat de dater la fabrication de la fausse monnaie qui n'a pas l'obligation - comme la monnaie officielle - de porter lors de son émission l'effigie du prince régnant. L'habitude s'est prise de dater cette activité en fonction du moule le plus récent qui ne fournit, bien évidemment, à l'instar de la dernière monnaie d'un trésor, qu'un terminus post quem, à cette différence près que la date réelle de fabrication d'un moule peut être bien éloignée de la date de l'original qui a fourni l'empreinte. En effet, le faussaire pouvait utiliser aussi bien des monnaies en circulation que des espèces thésaurisées, l'essentiel étant qu'il puisse les écouler et qu'il y trouve un profit.

A cet égard, le moule $n^{0} 3$ de Corseul offre ceci de remarquable qu'il porte à la fois l'empreinte d'un droit de denier de Julia Domna des années 196-211 et celle du revers d'un antoninianus attribuable à Aurélien (fig. 1), émis par la troisième officine de l'atelier de Milan entre la fin de 271 et l'été $273^{\text {? }}$.

De tels écarts de date sont rarement relevés dans les trouvailles de moules (cinq cas en France ${ }^{8}$,

6 J.-P. Callu, La politique monélaire des empereurs romains de 238 à 311, Paris, BEFAR 214, 1969, p. 259-260.

7 Il n'est pas possible de trancher entre la deuxième et la troisième émission, la distinction s'exerçant essentiellement sur l'évolution stylistique du portrait d'Aurélien.

8 Bordeaux (Gironde) : 4 moules de Caracalla à Postume; Damery (Marne) : 300 moules dont 32 intacts de Caracalla à Postume et Quintille (?); Châteaubleau (Seine-et-Marne) : 200 moules de Julia Domna à Tetricus; Rezé (Loire- 
un en Angleterre ${ }^{9}$ ) et. toujours sur des moules différents, ce qui permettait d'envisager une production de longue durée. En règle générale, les découvertes semblent plus homogènes, essentiellement sévériennes (193-235) avec quelques prolongements sous Maximin et Gordien III (238-244), voire Philippe (244-249), Mais il est vrai que nous souffrons d'un manque de publications de ce type de matériel ${ }^{10}$, et qu'il n'est donc pas assuré que toutes les empreintes découvertes - notamment les revers - aient été correctement déchiffrées. Or le moule de Corseul fournit la preuve, cuite dans l'argile, d'une fabrication de deniers coulés sous Aurélien, à partir d'originaux frappés quelque soixante ans plus tôt.

Cette constatation invite à s'interroger sur la date et la signification de cette industrie "coupable": s'agit-il d'une activité étalée dans le temps, du II $^{\mathrm{e}} \mathrm{s}$. jusqu'à la Tétrarchie, en quelque sorte le reflet d'un goût chronique de la malversation? Les auteurs sont-ils des faux-monnayeurs tirant profit des désordres monétaires ou des fonctionnaires locaux "s'ingéniant à lutter contre une certaine pénurie de numéraire " 11 ?

Cette dernière opinion a les faveurs de l'«école française $)^{12}$ qui développe une argumentation basée

Atlantique) : au moins 52 moules de Tibère et Vespasien à Maximin et Probus; Melun (Seine-et-Marne) : plus de $50 \mathrm{mou}-$ les de Caracalla à Dioclétien? (documentation inédite G. Aubin). Pour la bibliographie, voir M. Jungfleisch, J. Schwartz, Les moules de monnaies impériales romaines (essai bibliographique), Supplémenl aux Annales du Service des Antiquités, Cahier no 19, Le Caire, 1952, 35 p.; - J. Schwartz, Supplément à la bibliographie des moules de monnaies impériales romaines, Gazelle Numismatique Suisse, 13, nov. 1963, p. 12-14; - J.-P. CALlu, La politique ..., op. cil., p. 258260 et p. 456.

9 G.-C. Boon, P.-A. Rantz, Third Century Counterfeiting at Whitchurch, Somerset, The Archaeological Journal, CXXII, 1965 [1966], p. 13-51 (Aes d'Hadrien et de Sévère, antoniniens de Macrin et de Gallien à Tetricus). Une liste des découvertes britanniques est. donnée par G.-C. Boon, Counterfeit Coins in Roman Britain in : P.-J. Casey, R. Reece (éd.), Coins and the Archaeologist, BAR 4, Londres, 1974, p. 95-171, tableau p. 111.

10 La France accuse un retard certain dans ce domaine. Les seules découvertes ayant donné lieu à un catalogue sont celles d'Autun (J.-G. Bulı.ot, Notice sur des moules de monnaies antiques trouvés à Autun, Mémoires de la Société Éduenne, XXV, 1897, p. 45-64), du Verbe Incarné à Lyon (cf. note 5) et de Vienne (A. Pricforier, Vienne antique, Roanne, 1982, p. 337-347).

11 J. Schwart7, Moules monétaires, Numismatique antique. Problèmes et méthodes (Actes du Colloque de Nancy, 27 septembre-2 octobre 1971), Annales de l'Est, Mémoire no 44, Nancy-Louvain, 1975, p. 207-209.

12 Essentiellement J.-P. Callu, J. Schwartz, R. TurCAN (cf. notes $6,11,5$ ). sur la datation des moules. Même si l'on admet que la production du denier par moulage ne s'arrête pas en 238 et qu'elle n'a rien d'anachronique jusqu'au milieu du III' ${ }^{\mathrm{s}}$., on situe les débuts de cette activité sous les Sévères, notamment sous le règne de Sévère Alexandre (222-235) marqué par une forte dégradation de l'aloi du denier qui prend "la couleur terne et plombée du mauvais billon ${ }^{13}$. Cette dévaluation encourageait les contrefaçons en métal vil. Par ailleurs cette période voit l'implantation de l'antoninianus, nouvelle espèce qui supplante le denier à partir de 238. Compte tenu de sa valeur nominale (1,5 ou plutôt 2 deniers), "on avait intérêt à couler des espèces à effigies radiées après $240 n^{14}$. L'essor de l'antoninianus provoquait en dehors de l'Italie une pénurie de l'espèce divisionnaire (le denier) et "c'est cette demande que venait combler, en partie, le mauvais métal des séries officieuses ${ }^{15}$. L'extrême rareté des imitations coulées de Tetricus à Probus s'expliquerait, selon J.-P. Callu, par le fait que l'imitation au moule, de qualité supérieure, "ce qui aide à la faire accepter" n'est pas de mise "dans les années de crise où l'on reçoit la monnaie, si éloignée soit-elle de son modèle primitif " ${ }^{16}$.

Les moules de Corseul, de par leur chronologie, m'amènent à envisager un schéma différent. Il convient en effet d'expliquer pourquoi en 271-273 ou postérieurement - on a jugé utile ou profitable de couler des deniers et qui plus est en reproduisant des monnaies vieilles de 60 ans.

Première interrogation : le denier circulait-il encore sous Aurélien? L'examen des trésors répond par la négative. M. Christol ${ }^{17}$ a montré comment les deniers qui forment en gros les deux tiers des espèces cachées sous Philippe (244-249) et sous Trajan Dèce (249-251) n'en représentent plus que $28 \%$ sous Trébonien Galle et Volusien (251-253), 10 à $25 \%$ en 256-258 pour tomber à $3 \%$ en 260 . Toutefois, le denier ne disparaît pas totalement des trésors même si sa présence est parfois symbolique (7 deniers contrc 4906 antoniniens dans le trésor de Rocquen-

13 R. Turcan, Le trésor de Guelma. Étude historique et monétaire, Paris, Éd. Arts et Métiers Graphiques, 1963, p. 48.

14 R. Turcan, Les moules ..., op. cit., p. 11.

15 J.-P. Callu, op. cit., p. 260.

16 Ibid., p. 260, note 2.

17 M. Christol, Effort de guerre et ateliers monétaires de la périphérie au urre siècle après $J$.-C. L'atelier de Cologne sous Valérien et Gallien, in: Armées et fiscalité dans le monde antique, Colloque national du CNRS, no 936 (Paris 1416 octobre 1976), Paris, 1977, p. 235-277. 
court enfoui en 266) ${ }^{18}$. Chacun a en mémoire certains gros trésors célèbres qui conservent encore au temps de Postume (Eauze, Talmont), de Probus (Plouhinec, Cunetio), voire de Constantin (Beaurains) une quantité non négligeable de vieux deniers thésaurisés. En effet, cette monnaie n'est plus émise depuis 240. Notons toutefois la frappe épisodique de deniers sous Philippe, Dèce, Galle, et plus tardivement sous Gallien et Aurélien. Certaines de ces frappes ont été considérées comme des essais d'aurei; on incline aujourd'hui à y voir des espèces distribuées lors des largesses impériales, tels les donativa ${ }^{19}$. Quoi qu'il en soit, le denier n'aura que peu d'importance dans la circulation des années 270-274. Retenons plutôt à son propos la notion de thésaurisation.

En effet, quelle pouvait être à cette époque la relation de valeur entre le denier et l'antoninianus? Il est vraisemblable que le taux originel de change ( 1 antoninianus $=1,5$ ou 2 deniers) n'était plus en vigueur depuis longtemps. Comment en aurait-il pu être autrement? Sous Caracalla, le denier a un poids de fin d'environ $1,48 \mathrm{~g}$ et l'antoninianus de $2,38 \mathrm{~g}$ (mais déjà de $1,88 \mathrm{~g}$ sous Gordien). Par la suite, le titre ne cesse de se dégrader pour tomber de 440$430 \%$ à $20-30 \%$ sous Aurélien. En d'autres termes, l'ancien denier sévérien contenait environ dix fois plus de métal précieux que le billon d'Aurélien. Dès lors, les particuliers avaient intérêt à conserver précieusement, les deniers anciens accumulés en raison de leur valeur intrinsèque, voire à les rechercher. D'ailleurs, le Prince - Dèce puis Galle - avait procédé à leur retrait systématique en vue de leur refonte ${ }^{20}$. On est donc tenté de croire que le denier

18 D. Hollard, P. Gendre, Le trésor de Rocquencourt et la transformation du monnayage d'imitation sous le règne de Postume, Trésors Monétaires, VIII, 1986, p. 9-45, pl. I-XXIII.

19 J.-P. Gallu, op. cit., p. 257-258. Sur les émissions de deniers de Gallien et d'Aurélien, on consultera : J. Gricourt, Le trésor de Bavai (Nord), in: Trésors monétaires el plaques boucles de la Gaule romaine: Bavai, Montbouy, Chécy, $12^{\mathrm{e}}$ suppl. à Gallia, 1958, p.11-13; - P. Bastien, H.-G. Pflaum, Trouvaille de monnaies romaines de Thibouville (Eure), Gallia, 20 , 1962, p. $277 s q . ;-J$. Lafaurie, Réformes monétaires d'Aurélien et de Dioclétien, Revue Numismatique, 17, 1975, p. 95-98 (récuse le terme de denier et propose d'y voir le semis de l'aurelianus); - S. Estiot, Le trésor de Maravielle (Var), Trésors Monétaires, V, 1983, p. 37-39; - P. BAstien, Pseudoépreuves d'aurei et essais dans le monnayage impérial romain, Mélanges offerts au docteur J.-B. Colbert de Beaulieu, Paris, 1987, p. 77-87; - S. Estror, Un denier de Tétricus II provenant du trésor de Sainte-Pallaye, Bulletin de la Société Française de Numismalique, novembre 1988, p. 459-461.

20 .J.-P. C.ALlu, op. cit., p. 257. Pour les analyses, on se reportera à cet ouvrage. avait acquis un cours parallèle et officieux, croissant au fur et à mesure de la dégradation de l'antoninianus.

Dans cette perspective, l'hypothèse d'un faux monnayage d'origine privée me paraît plus convaincante que celle d'une industrie officieuse, tolérée voire encouragée par les pouvoirs publics. Le denier sévérien - même imité - pouvait-il en 270 être considéré comme une monnaie divisionnaire? D'ailleurs, la disette monétaire qui s'amorce à la fin du règne de Postume (269) et connaît son maximum sous Aurélien et Probus fut palliée par d'énormes masses d'imitations radiées, essentiellement à l'effigie de Tetricus ${ }^{21}$. Le profit du faussaire est évident. N'a-t-il pas joué sur l'attachement à une espèce quasiment disparue et considérée dès lors comme réserve de valeur? Le faux denier qu'il écoulait avait d'autant plus de chance d'être accepté que la comparaison avec de vrais deniers était devenue difficile. En outre, rappelons l'analogie d'aspect entre les deniers plombeux de Sévère Alexandre et les monnaies coulées avec un alliage composé de cuivre, d'étain et de plomb. Le coulage offrait enfin des produits bien venus (effigies, types et légendes) d'un aspect supérieur au mauvais billon officiel de Gallien ou de Tetricus, a fortiori des imitations radiées d'exécution souvent maladroite. Mais pourquoi, dira-t-on, prendre la peine de couler des monnaies, procédé long et peu rapide, à une époque où les imitations radiées commencent à inonder le marché? On a voulu voir dans les monnaies coulées et les imitations frappées deux manifestations successives s'excluant. Il y aurait un temps pour le faux coulé et un temps pour le faux frappé. En fait, rien ne s'oppose à ce que les deux phénomènes aient coexisté surtout si l'on veut bien admettre qu'ils n'ont ni les mêmes auteurs, ni le même but, ni la même clientèle.

D'un côté, des autorités tolèrent ou encouragent une production massive d'imitations radiées véritable monnaie de nécessité - pour procurer des moyens de paiement au plus grand nombre. De l'autre, des faussaires - qui utilisent aussi la technique de la frappe notamment sous

21 J.-B. Giard, La monnaie locale en Gaule à la fin du III $^{e}$ siècle, reflet de la vie économique, Journal des Savants, 1969 , p. 5-34, 2 pl. ; - G. AubIN, Trésors et imitations radiées dans l'Ouest de la Gaule, Bulletin de la Société Française de Numismatique, décembre 1982, p. 251-254; - D. Holla RD, Le trésor de Rouilly-Sacey (Aube), Trésors Monétaires, IX, 1987, p. 53-91, pl. XII-XV. 
Postume ${ }^{22}$ - s'assurent des profits certains en coulant avec soin des monnaies anciennes à l'intention de thésaurisateurs nostalgiques soucieux de préserver leur épargne en monnaie réputée bonne (qu'il s'agisse de deniers sévériens ou d'antoniniens de la bonne époque des Gordien et des Philippe) visà-vis de la monnaie des princes régnants dont on pouvait mesurer à l'œil nu l'avilissement. Il arrivait que ces épargnants avisés se fassent berner et qu'en essayant de réunir d'anciens deniers qui n'avaient plus cours légal mais conservaient une valeur dans un marché parallèle, ils aient reçu des faux fraîchement coulés sans valeur intrinsèque. Toutefois, l'ampleur du phénomène ne doit pas être surestimée. Le nombre de trésors comportant des monnaies coulées est peu important. Certes, jusqu'à unc date récente, les numismates n'ont pas été plus avisés que les thésaurisateurs antiques et n'ont pas décelé les intrus. Mais voyons bien que ces trésors comportent aussi, en nombre variable, des imitations frappées qui ne pouvaient guère tromper. Nuançons donc notre appréciation sur la perspicacité des usagers antiques. Il est possible que la désorganisation de la circulation circa 268-290 les ait conduits à accepter nolens, volens tout moyen de paiement sachant qu'à leur tour ils pourraient l'utiliser. Plus que jamais, il devient nécessaire de différencier trésors de thésaurisation et trésors de circulation ou plutôt dans un même trésor la part de la thésaurisation à laquelle s'ajoute le numéraire disponible au moment de l'enfouissement.

Faut-il aller plus loin et envisager de rajeunir les lots de moules sévériens, c'est-à-dire de privilégier la période 265-305 comme celle d'activité intense des faussaires? Il est bien évidemment impossible de répondre pour l'instant à cette question. On notera seulement que dans la mesure où le denier était l'espèce de prédilection des faussaires, il est logique que la plupart des lots de moules ne comportent pas d'empreintes postérieures à Gordien. La validation de cette hypothèse nécessiterait trois démarches complémentaires :

- la publication systématique des moules conservés dans les musées;

- le relevé dans les trésors des monnaies coulées, ce qui fournira des indices sur leur date de fabrication, antérieure à la dernière monnaie de l'enfouissement;

22 D. Gricourt, D. Hollard, L'articulation des frappes de bronze et de billon dans la production de l'atelier II sous Postume, Cahiers Numismatiques, 93, 1987, p. 302-314.
- la datation par archéomagnétisme des moules $^{23}$

Un dépouillement rapide des éditions récentes de trésors monétaires ne m'a fourni à ce jour que peu d'indications.

Courcité (Mayenne) ${ }^{24}$.

Enfoui en 270-271. Sur 3258 antoniniani, 116 imitations frappées et 8 monnaies coulées (Gordien III : 2; Otacilie : 1; Trajan Dèce : 1; Trébonien Galle : 1 ; Postume : 3 ).

Marboué (Eure-et-Loir) ${ }^{25}$.

Enfoui fin 273-274. Sur 1684 monnaies, 27 imitations dont 3 coulées (Gallien : 1 ; Postume : 2).

Bourg-Blanc (Finistère) ${ }^{26}$.

Enfoui en 276-277. Sur 826 monnaies, un denier coulé de Caracalla, dont le prototype date de 216 .

Rouilly-Sacey (Aube) ${ }^{27}$.

Enfoui postérieurement à 278. Sur 3598 monnaies, 3032 imitations dont 10 coulées (Gallien : 2; Victorin : 2; hybride Victorin/Gallien : 1 ; Tetricus : 5).

Bus-la-Mésière (Somme) ${ }^{28}$.

Enfoui vers 278. Sur 782 monnaies, 70 produits irréguliers dont une seule coulée (Tetricus II).

Thibouville (Eure) ${ }^{29}$.

Enfoui en 298. Sur 3256 monnaies, une coulée (Tetricus II).

23 Une première tentative réalisée à ma demande par $\mathrm{Ph}$. Lanos (Laboratoire d'Archéométrie de l'Université de Rennes I) sur un moule de Caracalla provenant de Melun apparaît peu concluante. D'autres essais sont nécessaires.

24 G. Aubin, Le trésor de Courcité (Mayenne) : antoniniani et imitations de Gordien III à Victorin, Trésors Monétaires, XI, 1989 p. 55-77, pl. XVII-XXXII.

25 M. Amandry, D. Hollard, S. Estiot, Le trésor de Marboué "Thuy" : antoniniens de Gordien III à Aurélien, in : Monnaies et trésors en pays Dunois, Bulletin de la Société Dunoise, XIX, 1985-1986, p. 45-69, pl. VI-XI (catal. nos 1682 1684).

26 M. Amandry, R. Sanquer, Le trésor de BourgBlanc (Finistère), Archéologie en Bretagne, 38, 1983, p. 15-46, pl. I-IV (catal. no 1 ).

27 Cf. note 21.

28 J. Gricourt, Le trésor du iII siècle de Bus-laMésière, Revue Belge de Numismatique, C, 1954, p. 31-56, pl. II-III.

29 P. Bastien, H.-G. Prlaum, Le trésor de monnaies romaines de Thihouville (Eure), Gallia, 19, 1961, p. 71-104; 20, 1962, p. 255 à 315 (catal. no 892). 
En Angleterre, le trésor de Cunetio ${ }^{30}$, riche de 54951 monnaies et peut-être caché en deux fois (27071 et 274-75) comporte 64 cast forgeries $(0,12 \%)$ contre 2085 siruck forgeries $(3,79 \%)$. Il s'agit surtout de copies d'antoniniens de Maximin à Tetricus et seulement de deux deniers (Balbin-Gordien III).

Bien évidemment, il convient surtout de déceler les trésors les plus anciens contenant des monnaies coulées $^{31}$. Pour l'instant aucun n'est antérieur à $270^{32}$.

Le dossier reste donc ouvert. Au total, ce petit

30 E. Besly, R. Bi.and, The Cunetio Treasure: Roman Coinage of the Third Century A.D., Londres, 1983, 199 p. 40 pl. Neuf autres trésors enfouis entre 274 et 296 en Bretagne romaine ont livré des monnaies coulées (R. Bland, A. Burnetr, The Normanby Hoard, Coins Hoards from Roman

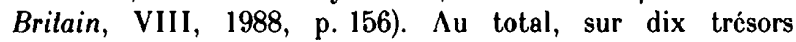
britanniques, 238 monnaies coulées ont ainsi été mises en évidence ce qui représente $0,17 \%$ des monnaies cachées et confirme le caractère marginal de cette production.

31 On notera que d'importants trésors comportant deniers et antoniniani enfouis entre 253 et 255 n'en contiennent pas. P. Le Gentilhomme, La trouvaille de Nanterre, document auquel on pourra reprocher son caractère isolé attire l'attention sur la datation d'un phénomène, certes marginal, mais qui contribue à la compréhension des attitudes, encore mal connues, de l'usager antique de la monnaie.

\section{Gérard Aubin}

Revue Numismatique, 1946, p. 15-114, pl. II-IV (219 deniers de Septime Sévère à Gordien III et 1643 antoniniani de Gordien à Gallien, règne conjoint); - H.-G. PFi.Aum, H. Huvfi.ın, Le trésor de Viuz-Faverges, Trésors Monétaires, 3, 1981, p. 33-76 (1 778 deniers de Néron à Gordien III et 525 antoniniens de Galle à Volusien. Ajouter deux deniers fourrès de Sévère Alexandre et Maximin).

32 Un moule à l'effigie de Septime Sévère a été découvert dans un contexte de la seconde moitié du IIre $\mathbf{s}$. lors des fouilles de la verrerie de Trinquetaille, en 1982-84 (Du nouveau sur l'Arles antique, Revue d'Arles, 1, 1987, p. 87 $\mathrm{n}^{\circ}$ 199). C. Brenot, Bulletin de la Société Française de Numismatique, 45, 1990, p. 719-721.

N.B. - L'illustration est due à M. Ch. Hémon, Musées départementaux de Loire-Atlantique, Musée Dobrée, Nantes. 